\title{
Historia y escatología en el Liber floridus de Lamberto de Saint-Omer
}

\author{
José Miguel de Toro \\ UNIVERSIDAD CATÓLICA DE LA SANTÍSIMA CONCEPCIÓN \\ jimdetoro@ucsc.cl
}

Resumen: El presente artículo tiene por objetivo analizar el corpus de textos históricos que Lamberto de Saint-Omer incluyó en su enciclopedia titulada Liber floridus (1121). Con la ayuda de los manuscritos Gantes, Bibl. Univ., 92 (autógrafo); Wolfenbüttel, HAB, Gud. Lat. 1; y París, BNF, lat. 8865, se propone una clasificación de los capítulos de carácter histórico y se muestra su conexión con el fondo espiritual general que impregna toda la obra. Lo anterior revela que Lamberto se sirve especialmente de la historia profana y del saber cosmográfico para nutrir su reflexión escatológica, y muestra cómo los sucesos de su época, en particular el éxito de la Primera Cruzada, estimularon en este autor el desarrollo de las ideas apocalípticas.

Palabras clave: Lamberto de Saint-Omer, Liber floridus, escatología, crónicas, Primera Cruzada, enciclopedismo, cosmografía.

Abstract: This article aims to analyze the corpus of historical texts that Lambert of Saint-Omer included in his encyclopaedia entitled Liber floridus (1121). Using as our basis the manuscripts Ghent, Univ. Lib., 92 (autograph); Wolfenbüttel, HAB, Gud. Lat. 1; and Paris, BNF, lat. 8865, we propose a classification of the historical chapters of the Liber floridus and also to show their connection with the general spiritual background that permeates the whole work. This reveals that Lambert uses especially profane history and cosmographic knowledge to nourish his eschatological reflection, and shows how the events of his time, particularly the success of the First Crusade, encouraged the author to develop apocalyptic ideas.

Keywords: Lambert of Saint-Omer, Liber floridus, eschatology, chronicles, First Crusade, encyclopaedism, cosmography. 


\section{INTRODUCCIÓN ${ }^{1}$}

El Liber floridus de Lamberto, canónigo de la Iglesia Notre-Dame de Saint-Omer, en Flandes, es un texto de carácter enciclopédico terminado en 1121. Elaborado siguiendo el método de la compilación, se presenta bajo el formato de un extenso volumen de casi doscientos capítulos, que incluyen comentarios filosóficos y teológicos, crónicas, vidas de santos, descripciones del mundo y del sistema planetario, además de catálogos de plantas, animales, piedras y un sinfín de otros tópicos². Dentro del ingente material reunido por Lamberto, destacan tres tipos de textos: los cosmográficos, los teológicos y los históricos. Entre los primeros encontramos una amplia variedad de extractos tomados de cosmógrafos de la Antigüedad Tardía, especialmente de Isidoro de Sevilla, Marciano Capela, Macrobio y Ético Íster ${ }^{3}$, complementados con esquemas y diagramas diseñados por el propio canónigo, que le permiten ejemplificar el sistema de las esferas planetarias, la posición de los astros (en especial los signos del zodiaco), fenómenos celestes como los eclipses, y representaciones cartográficas (mapamundis, mapas locales y planos de ciudades).

En el ámbito teológico, es la escatología la que acapara la atención de Lamberto, con un buen número de comentarios que invitan a la contemplación de los últimos días con consideraciones sobre el Apocalipsis, los novísimos y la venida del Anticristo. Esto confiere a esta peculiar enciclopedia un marcado sentido espiritual, por cuanto tiene

1 Este artículo forma parte del proyecto de investigación FONDECYT de iniciación $\mathrm{n}^{\circ}$ 11160240, de CONICYT, Gobierno de Chile, titulado "Renovación de saberes en el Occidente medieval: la influencia del enciclopedismo en la escritura de la historia durante el siglo XII".

2 A. Derolez, The Making and Meaning of the Liber Floridus. A Study of the Original Manuscript, Ghent, University Library MS 92 (Harvey Miller Publishers, LondonTurnhout 2015). Y del mismo autor, The Autograph manuscript of the Liber Floridus. A Key to the Encyclopedia of Lambert of Saint-Omer (Brepols, Turnhout 1998).

3 P. Gautier-Dalché, "Guillaume de Conches, le modèle macrobien de la sphère et les antipodes: antécédents et influence immédiate", en B. ОвRISт (dir.), Guillaume de Conches: Philosophie et science an XIIe siècle (SISMEL / Edizioni del Galluzzo, Florencia 2011) 229-231. Y del mismo autor, "Les 'quatre sages' de Jules César et la 'mesure du monde', selon Julius Honorius: La tradition médiévale”, en Géographie et culture. La représentation de l'espace du VIIe au XIIe siècle, (Ashgate, Aldershot-Brookfield 1997) 203-204. 
como objetivo presentar el mundo como el escenario donde opera la evangelización ${ }^{4}$. Los segmentos dedicados a esta temática son variados y ricos, recogiendo la tradición teológica de la materia iniciada en los siglos anteriores ${ }^{5}$ : Prognosticon futuri saeculi, De nominibus Sybillarum X, De paradiso et insulis, De paradysi fluminibus, De terre motu signisque diversis, De duobus paradisis, De excidio Iherusalem signa, De duobus infernis, De quodam Antichristi membro, De electis et reprobis, De signis in sole et luna et reliquis, Methodius de principio et fine, De Antichristo siginsque XV, y finalmente De adventu Domini in die iudicii. $\mathrm{El}$ autor elabora incluso una versión gráfica (Apocalypsis depictus), y dedica unas ilustraciones magníficas y sobrecogedoras a algunas figuras ligadas al fin de los tiempos, como Behemot, el Leviatán, el Anticristo y el Dragón infernal, añadiendo breves comentarios con el fin de explicar el rol que jugarán en ese proceso.

Junto a este conjunto de textos escatológicos aparecen los textos del tercer grupo, es decir, los pasajes relativos al pasado de la humanidad. En efecto, el Liber floridus se muestra especialmente pródigo en materia de historia universal, clásica y europea. Lamberto decidió incluir resúmenes de crónicas y confeccionó diversos recuentos temporales. Por sobre todo destaca una crónica, compuesta por él mismo, que abarca desde la creación del mundo hasta 1120, con una estructura narrativa primero, que luego muta en el esquema analístico. Por eso T. Reuter lo ubica dentro del movimiento de florecimiento del género cronístico a finales del siglo XI y principios del XII en el imperio germánico, emparentado con cronistas como Frutolfo de Michelsberg († 1103) y Otón de Freising († 1158) ${ }^{6}$.

La información de carácter histórico está repartida a lo largo de todo el Liber floridus, formando capítulos más o menos extensos de acuerdo a su contenido específico. Como la obra aún no ha sido objeto de una edición crítica, para su análisis hemos utilizado el manuscrito de

4 P. Gautier-Dalché, "De la glose à la contemplation. Place et fonction de la carte dans les manuscrits du haut Moyen Âge", en Géographie et culture..., 744-745.

5 J. T. Palmer, The Apocalypse in the Early Middle Ages (Cambridge University Press, Cambridge 2014).

6 T. Reuter, "Past, Present and No Future in the Twelfth-Century Regnum Teutonicum", en P. Magdalino (dir.) The perception of the past in Twelfth-Century Europe, (Hambledon Press, London-Rio Grande 1992) 33. 
Gantes, Biblioteca Universitaria, 92, siglo XII, autógrafo $(\mathrm{G})^{7}$. Como complemento a este manuscrito, lamentablemente mutilado en algunas secciones, se han usado los ejemplares de Wolfenbüttel, Herzog-AugustBibliothek, Gud. Lat. 1, segunda mitad del siglo XII (W), y de París, Bibliothèque Nationale, lat. 8865, del tercer cuarto del siglo XIII (P). Aunque el manuscrito $\mathrm{W}$ no contiene el Liber floridus en su totalidad, sino que llega hasta el capítulo CXXVI (Gesta Romanorum pontificum), sí presenta la lista completa de capítulos al comienzo del documento (folio 1r-v), lo que permite corroborar la estructura del Liber. En la copia P, en cambio, no aparece el índice propiamente tal pero el texto se encuentra en forma integral ${ }^{8}$. Nos hemos apoyado igualmente en el completísimo artículo descriptivo publicado por Léopold Delisle a principios del siglo pasado en el boletín Notices et extrait des manuscrits de la Bibliothèque Nationale y en la obra del gran estudioso belga del Liber floridus, Albert Derolez ${ }^{10}$.

En total, podemos contabilizar 57 capítulos de contenido histórico. Algunos de ellos no figuran en el índice del Liber pero forman unidades independientes en el cuerpo del texto y cuentan con su propio acápite. Todo ese material puede dividirse en cinco grupos textuales bien definidos. En primer lugar, aquellos capítulos que tratan de la historia y cronología universales, es decir, el recuento del pasado humano desde la creación del mundo hasta la época de Lamberto, incluyendo los imperios y reinos de

7 Solo contamos con dos ediciones facsimilares, la de Albert Derolez para el manuscrito de Gantes y la de Christian Heitzmann con Pratricia Carmassi para el manuscrito de Wolfenbüttel. Hay ediciones de distintos fragmentos en la colección Monumenta Germaniae Historica (MGH). Véase A. Derolez (ed.), Lamberti S. Audomari canonici, Liber Floridus. Codex autographus bibliothecae Universitatis Gandavensis (Gantes, E. Story-Scientia 1968); C. Heitzmann - P. Carmassi (ed.), Der Liber Floridus in Wolfenbüttel. Eine Prachthandschrift über Himmel und Erde (Darmstadt, Wissenschaftliche Buchgesellschaft 2014).

8 Este códice solo presenta una tabla de materias de todos los contenidos del manuscrito, adición del siglo XIV.

$9 \quad$ L. Delisle, "Notice sur les manuscrits du «Liber floridus» de Lambert, chanoine de Saint-Omer", en Notices et extraits des manuscrits de la Bibliothèque Nationale et autres bibliothèques 38 (1906) 577-791.

10 A. Derolez, The Making and Meaning..., 45-172. 
la antigüedad ${ }^{11}$. En segundo lugar, están los textos relativos a la historia pagana clásica greco-romana, con especial énfasis en el Imperio romano ${ }^{12}$. Otro grupo lo constituyen los capítulos de historia bíblica, donde los antiguos judíos tienen gran protagonismo. Este es el grupo más numeroso, aunque no por mucha diferencia ${ }^{13}$. En cuarto lugar, tenemos el grupo de secciones relativas a la historia de la Iglesia en general. Consideramos aquí también las vidas de santos, que son relativamente frecuentes en el Liber floridus $^{14}$. Si bien se han presentado serios reparos para considerar a la hagiografía como parte del género historiográfico en la Edad Media, la

11 Los capítulos correspondientes a este grupo son los siguientes: IV. Círculo de los soberanos que han gobernado, por edades; VI. Círculo del mundo y microcosmos; VII. Freculfo, sobre los gobernantes de los reinos; XVIII. Crónica de Isidoro; XIX. Sobre las seis edades [del mundo]; XXI. De nuevo, sobre las seis edades del mundo; CXXIV. Genealogía del mundo; CXXXV. Crónica del mundo de Orosio; CLXII. Sobre el árbol bueno y el malo; CLXIII. Sobre las seis edades del mundo, con los días comparados (capítulo desaparecido en el ms G); CLXXVII. Sobre la diversidad de los distintos tiempos; CLXXXVII. Sobre la genealogía del mundo; Sobre el inicio del mundo y Adán (no listado en el índice; ms G, ff. 154v-155v).

12 Los capítulos pertenecientes a este grupo son: V. Círculo del Minotauro de la casa de Dédalo; XXVIII. Sobre cinco ciudades famosas (capítulo desaparecido en el ms G); CXXXII. Sobre Alejandro Magno; CXXXIII. Sobre la caída del Imperio Romano, en verso; Hazañas de Cayo Julio César (no listado en el índice; ms G, f. 190v); CXXXVII. Hazañas de los emperadores romanos; CLXXXVIII. Hazañas del rey Apolonio; CXC. Sobre la caída de Troya, según Dares; Sobre los reyes y cónsules romanos, y sus guerras (no listado en el índice; ms G, f. 278v).

13 Los capítulos de este grupo son: II. Sobre los jueces de los judíos; VIII. Sobre los jueces de los judíos, según Egesipo; X. Sobre Salomón y su gloria; LXIII. Sobre la caída del pueblo de los judíos; LXXXVIII. Sobre Alejandro e Hircano; CXIII. Sobre Jacob y Esaú; CXXVII. Sobre la infancia de Moisés; CXXVIII. Testimonio de Josefo sobre Cristo; CXXIX. Sobre Herodes y su hermano Arquelao; CXXXVIII. Sobre el arca de Noé y los animales; CLXV. Sobre Adán y sus hijos, y sobre Babilonia (solo en el índice de W); CLXVII. Sobre de qué estirpe provino Herodes (capítulo desaparecido en el ms G); CLXVIII. Sobre los últimos soberanos de los judíos; CLXXI. Genealogía de Jesucristo.

14 Los capítulos de este grupo son: XXIV. Nombres de los pontífices romanos; LXI. Sobre los milagros en los Diálogos de San Gregorio; XC. Sobre Juan Evangelista; XCI. Sobre los 12 apóstoles; CXIV. Sobre la Iglesia de Saint-Omer [y sobre san Bavón]; CXVIII. Conflicto entre Enrique y Pascual; CXXXVI. Hechos de los pontífices romanos; Nombres de los obispos de los francos (no listado en el índice; ms G, f. 240v); CLXXXIX. Sobre san Audomaro y los prepósitos del lugar; Nombres de abades (no listado en el índice; ms G, ff. 270v-271r). 
incluimos por tratarse de un modo de escritura asimilable a la biografía ${ }^{15}$. El último grupo textual corresponde a la historia europea, aquella que versa sobre los pueblos germánicos y los reinos medievales. Este grupo comprende igualmente los hechos de los europeos en las comarcas de Oriente durante el conflicto de las cruzadas ${ }^{16}$. El presente artículo tiene por objetivo analizar los segmentos relativos a la historia universal y profana, con el fin de entender en su contexto cómo ellos servían a un proyecto redaccional global donde se manifiesta una estrecha relación entre la escritura de la historia y la concepción escatológica propia de la época.

\section{El Marco Conceptual: La Historia de la Salvación}

Los capítulos correspondientes a la historia universal tienen como hilo conductor a los antiguos patriarcas, los reyes de los judíos y los emperadores (persas, griegos y, finalmente, romanos), es decir, siguen la estructura tradicional u ordinatio propia de las llamadas crónicas universales ${ }^{17}$. Sin explicitarlo en cada caso, Lamberto se basa en las obras de Paulo Orosio, Isidoro de Sevilla, Beda el Venerable, Helperico y Freculfo de Lisieux ${ }^{18}$, los principales cronistas de la Temprana Edad Media. A partir de ellos elabora pequeñas cronologías y recuentos de tiempo que sirven para

15 M. Aurell, "L'historiographie ecclésiastique en Occident (IVe -XIIe siècles): Providence, type, exemple", en L. MARTínez FerRer (dir.), Venti secoli di storiografia ecclesiastica. Bilancio e prospettive (EDUSC, Roma 2010) 58-71.

16 Los capítulos de este grupo son: XXII. Años [del mundo] desde el nacimiento del Señor Jesucristo (Anales); XXIII. Nombres de los reyes romanos; LVII. Historia de los anglos; LX. Genealogía de los condes normandos; Nombres de los condes de Flandes (no listado en el índice; W, f. 71v); CXVII. Genealogía de los condes de Flandes; CXX. Hazañas de los cristianos en Jerusalén; Genealogía de Oduino y del comandante Heimerico, su hijo (no listado en el índice; ms G, f. 154r); CXXXVII. Hazañas de los emperadores romanos (este capítulo figura también en el grupo de historia clásica, porque se refiere tanto a la historia de los emperadores romanos paganos como a sus continuadores cristianos hasta llegar a los emperadores del Sacro Imperio medieval); CLIII. Hazañas de los daneses, godos y hunos; CLV. Sobre la división de las provincias de los francos; CLXIX. Sobre los primeros reyes de los francos.

17 M. Chazan, "La méthode critique des historiens dans les chroniques universelles médiévales", en M. ChazAn - G. Dahan (dir.), La méthode critique au Moyen Âge, (Brepols, Turnhout 2006) 224.

18 A. Derolez, The Making and Meaning... , 40, 195-199. 
contextualizar, en el devenir general de la humanidad, los temas históricos particulares que va tratando en los otros capítulos.

Pero sobre todo, las crónicas universales permiten a Lamberto construir el soporte de una concepción histórica conocida como Historia de la Salvación. Este era el principal marco epistemológico en la escritura de la historia y provenía de la teología de san Agustín, quien había sentado las bases de lo que fue el esquema divisorio del tiempo en la Edad Media ${ }^{19}$. Para los intelectuales de la época, la Historia se componía de seis etapas marcadas por la acción de Dios en el mundo, que guía a la humanidad paso a paso desde la caída del pecado hasta su liberación definitiva. Por eso los hitos que dividían cada edad estaban tomados de la historia bíblica y estaban cargados de un fuerte significado teológico. La primera edad iba desde la Creación hasta el Diluvio; la segunda, del Diluvio hasta Abraham; la tercera, desde Abraham hasta la construcción del Templo de Jerusalén en tiempo del rey Salomón; la cuarta, desde Salomón hasta la cautividad en Babilonia; la quinta, desde la liberación de los judíos por Ciro hasta la venida de Cristo, quien marcaba el inicio de la sexta y última edad terrena. Con el fin de este mundo comenzaría la séptima edad de gozo con Dios en la Creación renovada.

El enorme éxito que tuvo esta concepción histórica tiene que ver, sin duda, con el prestigio del obispo de Hipona, pero también con el fuerte carácter alegórico que ella posee, algo muy acorde con la sensibilidad de la cultura teológica medieval. Seis días duró la Creación, seis edades debe recorrer la humanidad antes del descanso definitivo. A diferencia de la doctrina pagana clásica sobre la historia, el cristianismo heredó del pensamiento hebreo la noción fundamental de que el mundo tiene un principio claramente definido: la Creación ex nibilo, el punto de partida

19 Agustín de Hipona, De Genesi contra Manichaeos, 1, 23. D. Weber (ed.), Sancti Augustini Opera (CSEL 91; Verlag der Österreichischen Akadamie der Wissenschaften, Viena 1998); Enarrationes in Psalmos, Ps. 92, 1. E. DekKers - J. Fraipont (ed.), Aurelii Augustini Opera. Pars X, 2 (CCSL 39; Brepols, Turnhout 1990); De catechizandis rudibus, 22. J.B. Bauer (ed.), Aurelii Augustini Opera. Pars XIII, 2 (CCSL 46; Brepols, Turnhout 1969). Véase una exposición ordenada del pensamiento agustiniano sobre este particular en E. Baura, Aetatis mundi sunt... La división de la historia durante la Edad Media (siglos IV a XIII) (La Ergástula, Madrid 2012) 51-63. 
absoluto de la historia ${ }^{20}$. Este punto de partida remite, a su vez, a un final tan definido y absoluto como el principio, al menos en lo que se refiere a la existencia terrena, situación que formó parte de la predicación cristiana desde los comienzos ${ }^{21}$. Y pese a las advertencias de Cristo, los teólogos de la Antigüedad Tardía intentaron calcular la duración total de la historia, por ejemplo, a partir de lo que se dice en el Salmo 90,4: "Porque mil años a tus ojos son como el ayer, que ya pasó, como una vigilia de la noche", lo que es reforzado en la $2^{\text {a }}$ Carta de Pedro 3,8: "Pero ustedes, queridos hermanos, no deben ignorar que, delante del Señor, un día es como mil años y mil años como un día". Así, en concordancia con los días de la creación, el mundo debía durar 6.000 años.

No es extraño, pues, encontrar en el Liber floridus, el esquema de la historia universal puesto en relación con los días de la creación. Y Lamberto también lo vincula con las edades del ser humano, que es, de acuerdo a la doctrina cosmográfica clásica ${ }^{22}$, un mundo en miniatura o microcosmos. En el folio 20v del manuscrito de Gantes, bajo el esquema con las seis edades del mundo, Lamberto añade la siguiente analogía con la vida humana:

Microcosmos, esto es un mundo más pequeño, descrito a través de las siete edades del ser humano. Descripción de la edad del ser humano: Infancia hasta los 7 años. Puericia hasta los 14 años. Adolescencia hasta los 28 años. Juventud hasta los 50 años. Gravedad hasta los 70 años. Senectud hasta los 80 años. Decrepitud hasta el final de los años ${ }^{23}$.

20 M. WallrafF "Von der antiken Historie zur mittelalterlichen Chronik. Die Entstehung christlicher Universalgeschichtsschreibung", en Welt-Zeit. Christliche Weltchronistik aus zwei Jahrtausenden in Beständen der Thüringer Univesitäts- und Landesbibliothek Jena, (Walter de Gruyter, Berlín-New York 2005) 3-6.

21 E. Baura, Aetatis mundi sunt..., 40-41.

22 J. Le Goff - N. Truong, Una historia del cuerpo en la Edad Media (Paidós, Barcelona 2005) 130-131.

23 G, f. 20v: "Microcosmos, hoc est minor mundus, per VII ętates hominis descriptus. Descriptio ętatis hominis. Infantia usque ad annos VII. Pueritia usque ad annos XIIII. Adolescentia usque ad annos XXVIII. Iuventus usque ad annos L. Gravitas usque ad annos LXX. Senectus usque ad annos LXXX. Decrepita usque ad annos finis". 
En la mente de Lamberto, entonces, no puede hablarse de la sexta edad del mundo y de los acontecimientos que tienen lugar en su época, sin poner como telón de fondo el panorama completo de la historia humana. Cristo, en su segunda venida, recapitulará todas las cosas (Ef. $1,10)$ consumando la historia y poniendo fin a la peregrinación de los hombres. Al final del capítulo CXXIV (Genealogia mundi), que comprende un recuento de la historia universal, Lamberto agrega:

Al final de esta edad [la sexta] habrá una gran discordia y grandes signos. Y como hubiere sido revelado el hijo de la perdición, el Anticristo, reinará durante tres años y medio. Cuando haya sido muerto por el Espíritu de Dios vendrá el día del Señor y este mundo llegará a su fin ${ }^{24}$.

\section{La Antigüedad Pagana y la Escatología Cristiana}

Las secciones relativas a la antigüedad pagana se concentran especialmente en la historia de Grecia y Roma. Aquí resaltan dos elementos: el Imperio y Alejandro Magno. El primero tiene que ver con una institución política que representaba el pilar de la historia profana, aunque encauzado igualmente en la Historia de la Salvación. Lamberto conocía bien la interpretación que los Padres de la Iglesia habían hecho del libro de Daniel. Según ella, la estatua soñada por Nabucodonosor (Dn. 2) y las bestias salidas del mar (Dn. 7) representaban reinos paganos que iban sucediéndose en el tiempo y transmitiéndose el poder universal en una dirección este-oeste. Nacido en Babilonia, el corazón de Oriente, el imperio se fue trasladando hacia Occidente hasta llegar a su culmen en el Imperio Romano y Romano cristiano (translatio imperii), compenetrando la historia del mundo con la de la Iglesia, en lo que el obispo Otón de Freising, un contemporáneo de Lamberto, llamaba la civitas permixta ${ }^{25}$. Así pues, el Imperio representa también un elemento de tensión hacia el futuro, por cuanto tiene la misión, como estructura política querida por Dios, para organizar la

24 G, f. 139r: "Gravis dissensio in fine huius etatis et portenta magna erunt. Et cum revelatus fuerit filius perditionis Antichristus, regnabit tribus annis et dimidio. Quo Dei spiritu interfecto dies Domini veniet et mundus iste finietur".

25 Otón de Freising, Chronica, VII, Prólogo: "A tempore Theodosii senioris usque ad tempus nostrum non iam de duobus civitatibus, sed de una paene civitate, sed permixta historiam texuisse". A. HofmeIster (ed.), Ottonis Episcopi Frisingensis Chronica sive Historia de duabus civitatibus (MGH, SS rer. Germ., 45; Impensis bibliopolii Hahniani, Hannover-Leipzig 1912) 309. 
sociedad terrena y guiarla hacia su plenitud en el cielo, donde se fundirán sin mezcla de pecado la historia humana y la divina.

Alejandro Magno, por su parte, sirve al proyecto del canónigo de SaintOmer en un sentido muy específico. El material alejandrino, bastante abundante en el Liber floridus, consta de varias secciones. La primera es un breve resumen de la vida del caudillo, a partir del Epitome de Julio Valerio, estructurado en torno a los siguientes hitos: el rey Nectanebo y la infancia de Alejandro, la gesta militar en Oriente, el regreso a Babilonia y, finalmente, la muerte del conquistador en esta misma ciudad. Luego encontramos la Carta de Alejandro a Aristóteles, pletórica de maravillas y descripciones exóticas sobre la India; el intercambio epistolar entre Alejandro y Díndimo, rey de los brahmanes ${ }^{26}$; la fundación de las 12 Alejandrías; y, por último, una soberbia imagen a todo color de Alejandro cabalgando espada en mano sobre Bucéfalo. ¿Qué función cumple el hijo de Filipo en el discurso de la Historia de la Salvación? Una función crucial. La vida de Alejandro, rica en episodios fabulosos, posibilita la construcción de un escenario adecuado para los acontecimientos que tendrán lugar en el fin del mundo: en particular, la irrupción de los pueblos malditos de Gog y Magog, también conocidos como los pueblos inmundos (gens immunda).

El episodio del encierro de los inmundos no formaba parte de la historia alejandrina clásica. No obstante, Lamberto complementó esta con la información proveniente de un Comentario al Apocalipsis atribuido al obispo Metodio de Olimpia ( $†$ c.311) pero en realidad escrito hacia fines del siglo VII. Este texto, que pretendía dar un sentido a las conquistas musulmanas, está cargado de referencias proféticas y escatológicas ${ }^{27}$.

Lamberto lo sigue de cerca en muchos puntos. Incluso entrega un

26 Estos tres textos, a saber, el Epitome de Julio Valerio, la Carta de Alejandro a Aristóteles y la Collatio suelen encontrarse juntos en los manuscritos, y en forma independiente de la Historia de Alejandro. Véase D.J.A Ross, "A check-list of manuscripts of three Alexander texts: the Julius Valerius Epitome, the Epistola ad Aristotelem and the Collatio cum Dindimo", en Scriptorium 10 (1956), 127-132.

27 Contrariamente a lo que podría pensarse, la principal fuente bíblica utilizada por Pseudo Metodio no es el libro del Apocalipsis, sino que la base de su visión escatológica proviene de 2 Tes. 2, 1-12, y en menor medida del Libro de Ezequiel. B. Garstad (ed.), Apocalypse, Pseudo-Methodius. An Alexandrian World Chronicle (Harvard University Press, Cambridge (Mass.) - Londres 2012) xi. 
resumen de él en el capítulo CLVI (Methodius de principio et fine). De acuerdo al mismo, cuando el conquistador macedónico guerreaba en las inmediaciones del Mar Caspio encontró un pueblo de costumbres perversas y repugnantes. Con la ayuda de Dios logró encerrarlos entre las montañas, bloqueando la entrada con un grueso muro y una puerta de hierro, donde permanecieron hasta el presente multiplicándose. Al final de los tiempos, el Anticristo vendrá a liberarlos para que lo ayuden en su labor destructiva:

Como cuando estaban en los días de Noé, comiendo y bebiendo, alegrándose y casándose, y no tenían temor en sus corazones. Así será en la venida de Gog y Magog. Así, cuando hubieren encontrado la paz, entonces se abrirán las Puertas Caspias en los confines del Aquilón y aquellos pueblos que están en las regiones de Gog y Magog vendrán, y harán estremecer toda la Tierra por temor a ellos, y se espantarán todos los hombres que habitan sobre la faz de toda la tierra y se esconderán de su presencia en los montes y en las cavernas. Porque aquellos comen carnes de seres humanos y de serpientes y de animales de carga y mujeres con niños pequeños, y no habrá nadie que pueda someterlos. Durante siete años rodearán la ciudad de Josefés, y entonces el Señor enviará en su contra a uno de sus príncipes y los acabará con azufre y fuego de una sola vez. ${ }^{28}$

La reclusión de estos pueblos inmundos tiene un correlato gráfico en el Liber floridus puesto que aparecen señalados en un gran mapamundi. Si bien este mapa se ha perdido en el manuscrito autógrafo, es bien visible en los manuscritos de París $^{29}$ y Wolfenbüttel ${ }^{30}$, donde constituye el

28 G, f. 219r: "Cum vero fuerint in diebus Noę, ędentes et bibentes, letantes, nubentes, et non erit in corde eorum timor. Ita erit adventus Gog et Magog. Cum ita pax fuerit, tunc reserabuntur Portę Caspię in lateribus Aquilonis et gentes ille quę sunt in Gog et Magog venient, et concutietur omnis terra pre timore eorum, et pavescent omnes homines qui habitant super faciem tocius terrę et abscondent se in montibus et speluncis a conspectu eorum. Tales igitur carnes hominum comedent et serpentes et iumenta et mulieres cum parvulis, et non erit aliquis qui poterit expugnare eos. Per septem annos comprehendent civitatem Iosephen, et tunc immittet Dominus unum de principibus suis et percutiet eos cum sulphure et igne in momento uno". El relato original de Pseudo Metodio, en la versión latina, se encuentra en Comentario al Apocalipsis, 8; B. Garstad (ed.), Apocalypse, Psendo-Methodius..., 96-101.

29 W, f. 69v.

30 P, f. 62v. 
capítulo CX. En la imagen se aprecia una península en el extremo norte que da hacia el océano exterior y colinda con Europa, aunque separada de ella por el río Tanáis (el río Don). La península está unida al continente asiático por un estrecho paso en el cual las montañas ocupan todo el lugar, haciéndolo infranqueable. Allí se lee: "Aquí encerró Alejandro a los XXXII reyes" 31 , lo cual hace referencia a los pueblos malditos bajo la denominación de los XXII reyes (aquí modificados a treinta y dos) ${ }^{32}$.

Los pueblos malditos también figuran en el mapamundi que sirve de centro a un esquema planetario de tipo macrobiano, descrito en el capítulo LXXIX (Będa de astrología o De cursu solis et lune per signa XII). El mapa muestra los dos hemisferios, norte y sur, con un océano intermedio, rodeado de un esquema que representa las órbitas planetarias y los signos del zodiaco, todo flanqueado por los comentarios astronómicos del tratado De signis coeli del Pseudo Beda. En el hemisferio norte puede verse la misma península junto al Mar Caspio, cuyo paso terrestre está impedido por una cadena montañosa que hace el acceso (y la salida de ella) inviable, y el rótulo "Gog Magog"33. Esta vez, no obstante, la península aparece ubicada mucho más al este, confinando con la India.

Igualmente con ocasión de la gesta alejandrina, Lamberto brinda a los lectores dos ejemplos de sociedades eutópicas, que el caudillo encontró mientras realizaba sus hazañas. En primer lugar, el pueblo de los seres, que era el nombre con que los occidentales denominaban a los chinos desde la época romana. Ellos forman una comunidad donde reina la justicia perfecta, "donde se dice que no hay homicidio ni adulterio ni perjurio ni ebriedad; y se alimentan solo de pan, verduras y agua" ${ }^{34}$. Se trata, ciertamente, de una trasposición del clásico pueblo de los hiperbóreos,

31 W, f. 69v: "Hic inclusit Alexander regna XXXII"; P, f. 62v.

32 D. LeCoQ, "La Mappemonde du "Liber Floridus" ou La Vision du Monde de Lambert de Saint-Omer", en Imago mundi, 39 (1987), 27-28. La denominación de los 22 reyes proviene de Pseudo Metodio, Comentario al Apocalipsis, 8,10, donde identifica con nombre a 22 reyes que gobiernan entre los pueblos encerrados por Alejandro Magno.

33 G, f. $92 \mathrm{v}$.

34 G, f. 161r: "Gens iustissima omnium gentium esse perhibetur, ubi nec homicidium neque adulterium neque periurium neque ebrietas committi dicitur; pane tantummodo et holeribus et aqua vescuntur". 
bien conocido desde la antigüedad griega como una sociedad en la que no había mal alguno, ni físico ni moral ${ }^{35}$. Así se lee en un mapa confeccionado en la época de Lamberto conocido como Mapa de Sawley o Mapa de Enrique de Maguncia (Henry of Mainz): "Pueblo de los hiperbóreos, felicísimo, sin enfermedad ni discordia" ${ }^{\prime 36}$.

En segundo lugar, Lamberto introduce al pueblo de los brahmanes, también conocidos como gimnosofistas o filósofos desnudos. Según la leyenda, Alejandro entró en contacto directo con ellos y en particular con su rey Díndimo, con quien tuvo un fructuoso y elevado, aunque totalmente apócrifo, intercambio epistolar acerca de asuntos filosóficos, lo que dio origen a una obra anónima conocida como la Collatio Alexandri et Dindimi, cuya composición se remonta a los siglos IV y $\mathrm{V}^{37}$. Lamberto recoge parte de esta tradición y reproduce en el Liber algunos de los comentarios de Díndimo. El rey en su epístola explica que el pueblo de los brahmanes vive una vida simple, natural y libre del vicio de la lujuria. Tampoco padecen males físicos ni sociales:

No contamos ningún nombre ni género de enfermedades, sino que gozamos de las alegrías duraderas de una salud incontaminada. Por eso nadie entre nosotros hace uso de hierbas para sanar los cuerpos ni pedimos ayuda contra los males que aquejan a los otros. Nadie pide dinero a nadie, puesto que vivimos entre iguales. El lugar no engendra envidia puesto que nadie es superior a los demás. La igualdad de la pobreza nos hace a todos ricos ${ }^{38}$.

35 Se ve, por ejemplo, en el autor del siglo IV, Cayo Solino, Colección de hechos memorables, 16,1-6. F.J. Fernández Nieto (ed.), Colección de hechos memorables o El erudito (Gredos, Madrid 2001) 313-315.

36 "Gens Yperborea beatissima, sine morbo et discordia". Ms Cambridge, Corpus Christi College, 66, p. 2. Dicho sea de paso, en este mapa también aparece el encierro de Gog y Magog.

37 Capítulo 132; G, ff. 161v-162r. Véase M. Steinmann, "Some Remarks on the Manuscripts of the Collatio Alexandri et Dindimi: Addenda et corrigenda to Ross's Checklist of Alexander Texts", en Scriptorium 68 (2014), 102-110, donde se advierten los fragmentos de la Collatio contenidos en el Liber floridus.

38 G, 162r: "Hinc est quod nulla genera morborum numeramus et nomina, sed diuturnis gaudiis salutis intemeratę defruimur. Nullus itaque aput [sic] nos sanandis corporibus usus herbarum est nec in alienas pernicies auxilium petimus constituti. Opem non precatur alter ab alio, ubi vivitur inter pares. Locus non prebetur invidię, ubi nullus superior est. Omnes divites facit pauperitatis ęqualitas”. 
De esta manera, los seres y los brahmanes aparecen como dos modelos de perfección moral y social que ayudan a comprender el espacio místico de la salvación, prefigurando el estado de los bienaventurados, en un sentido anagógico. Constituyen, en definitiva, dos sociedades que representan el paraíso en la tierra; ejemplos paganos, ciertamente, del ya-pero todavía no de la teología cristiana (o de la tensión escatológica), y parte, a la vez, del horizonte onírico legoffiano en su vertiente cristianizada ${ }^{39}$.

Lo anterior marca un poderoso contrapunto con los monstruos que Lamberto describe en el capítulo CIII (De monstris et bimembris [sic]). A diferencias de las sociedades beatíficas, aquellos simbolizan más bien el pecado original y la deformación que este produce en la naturaleza humana y en la conducta. En este capítulo, el canónigo se hace cargo de las razas monstruosas e intenta explicar, con la ayuda de san Agustín y del Prognosticum futuri saeculi de Julián de Toledo ${ }^{40}$, el sentido de estas criaturas y sobre todo con qué forma van a resucitar en el último día. Pero no es cuestión solo de las características aparentemente contrarias a la naturaleza humana que experimentan los blemias, los cinocéfalos, los antípodas, los pigmeos oscuros, los panotios y otras razas monstruosas ubicadas en los márgenes del mundo ${ }^{41}$. También se listan en el capítulo los adoradores del sol en Oriente, los caldeos de Babilonia que dan culto a Belus, el inventor de la astrología (discipline syderalis inventor), los campasantes que viven desnudos, y los ictiófagos y trogloditas incivilizados (gens feras). Es decir, los males que aquejan a todos estos monstruos no son solo físicos sino también morales. Pese a todo, estas deformaciones no los hacen menos humanos y por tanto también están necesitados de salvación ${ }^{42}$.

39 J.M. De Toro, “Apud Indos ver est perpetuum. El extremo Oriente durante la Edad Media: una revisión del horizonte onírico de Jacques Le Goff”, en Revista de Historia 23/1 (2016) 76-77.

40 A. Derolez, The Making and Meaning..., 108.

${ }_{41}$ G, ff. 50r-51r; 53r.

42 Lo mismo se aprecia en la obra Wonders of the East escrita en inglés antiguo y muy difundida en la isla de Bretaña en el siglo XI, donde las razas monstruosas aparecen en forma organizada y jerarquizada. Esto con una intención soteriológica, es decir, el autor quería enfatizar que eran seres humanos y que podían ser salvados. G. Austin, "Marvelous peoples or marvelous races? Race and the Anglo-Saxon wonders", en T.S. Jones - D.A. Sprunger (dir.), Marvels, monsters and miracles. Studies in the medieval and early modern imaginations (Kalamazoo, Michigan 2002) 42-48. 
Por eso Lamberto concluye este apartado con las palabras de san Agustín:

En verdad, cualquiera que en cualquier lugar nazca ser humano, es decir, animal racional mortal, por insólita que sea para nuestros sentidos la forma de su cuerpo o su color o su movimiento o su sonido, ningún fiel cristiano debe dudar que tiene su origen en aquel único primer hombre ${ }^{43}$.

De esta manera, la teratología del Liber floridus tiene una conexión profunda con el problema del pecado y de la resurrección, y, en último término, con el misterio escatológico.

No hay que perder de vista que el rol de Alejandro Magno en la historia se explica a partir de una visión moralizante del mundo. Visión elaborada por el teólogo Rábano Mauro en la época carolingia († 856), uno de los autores utilizados por Lamberto ${ }^{44}$. Según Elizabeth Keen ${ }^{45}$, el intelectual carolingio estaba particularmente preocupado de las interpretaciones morales. Para él, el mundus también podría ser entendido historialiter, es decir, como un constructo narrativo en clave alegórica; y como una compactio o conjunto de elementos opuestos como el bien y el mal, que no es otra cosa que el desarrollo de la metáfora agustiniana de la vida como viaje desde la región de la desemejanza con Dios (regio dissimilitudinis) hacia la verdadera patria en unión con É $1^{46}$. Bajo esta óptica cobran sentido las sorprendentes proezas de Alejandro Magno (y de otros viajeros legendarios como Noé, Odiseo y Bruto), en cuanto encarnaciones literarias de sus significados morales. Lamberto juzgó oportuno poner las hazañas alejandrinas a contribución de la escatología,

43 G, f. 53r : "Augustinus in libro XVI de Civitate Dei, capitulo VIII. Verum quisquis uspiam nascitur homo, id est animal rationale mortale, qualibet nostris inusitatam sensibus gerat corporis formam seu colorem sive motum sive sonum, ex illo uno protoplasto originem ducere nullus fidelium dubitet".

44 A. Derolez, The Autograph manuscript..., 198.

45 E. KeEN, "Shifting horizons: the medieval compilation of knowledge as mirror of a changing world", en J. KöNIG - G. WoOLF (dir.), Encyclopaedism from Antiquity to the Renaissance (Cambridge University Press, Cambridge 2013) 284-286.

46 Agustín de Hipona, Confessionum libri tredecim, 7, 10. L. Verhejen (ed.), Aurelii Augustini Opera. Pars I, 1 (CCSL 27; Brepols, Turnhout 1981). De ahí también las comparaciones que ofrece Lamberto entre lo viejo y lo nuevo, la Iglesia y la Sinagoga (G, ff. $231 \mathrm{v}-232 \mathrm{r})$. 
a fin de aportar antecedentes sobre los actores que tendrían un papel relevante en el momento del gran trance al final de los tiempos. Incluso ayudado de herramientas cartográficas puesto que, tal como ha mostrado Alessandro Scafi, los mapas medievales servían para espacializar las nociones escatológicas ${ }^{47}$.

La Historia de Apolonio, rey de Tiro, contenida en el capítulo CLXXXVIII, parece en cambio un texto completamente ajeno a una enciclopedia académica, como hacía notar Albert Derolez ${ }^{48}$. Se trata, en efecto, de una novela, un material más propio de un ciclo literario que de las sesudas disquisiciones computísticas, teológicas y filosóficas que abundan en el Liber. El mismo Lamberto es consciente de ello, puesto que después de incluir este relato, escribió una lista de personajes, al estilo de las dramatis personae propios de los textos literarios ${ }^{49}$. Pero la acción se desarrolla en Oriente y es ahí donde Lamberto tiene centrada su mirada, porque es en Levante donde están ocurriendo los acontecimientos más importantes del momento: la toma de Jerusalén por los cruzados y la construcción del reino latino. Las cruzadas, los grandes héroes de la antigüedad (Apolonio, Alejandro Magno), la irrupción de los pueblos malditos..., todo contribuye a decorar ese espacio donde aparecerán las primicias de la consumación de la historia. El hecho de que la Historia de Apolonio sea una novela no le impide cumplir esta función. Es más, el Liber floridus contiene material de todos los grandes ciclos épicos medievales, con excepción del Carolingio: Alejandro Magno, Troya, el rey Arturo, Apolonio de Tiro ${ }^{50}$.

Así se entiende también el capítulo dedicado a la caída de Troya (cap. CXC). Pues este hecho decisivo de la Antigüedad, muy valorado por los

47 A. SCAFI, "Mapping the End: The Apocalypse in Medieval cartography", en Literature and Theology 26/4 (2012) 205-411.

48 A. Derolez, The Making and Meaning..., 165.

49 Aunque Lamberto no es el primero en incluir material proveniente de un ciclo literario en una crónica. Frutolfo de Michelsberg a finales del siglo XI incorporó una gran porción de la Vita Alexandri Magni de Pseudo Calístenes (en la versión traducida por León de Nápoles) en su crónica universal. Más adelante también, el cronista francés Ricardo de Poitiers incluyó en su crónica pasajes tomados de la Historia regum Britanniae de Godofredo de Monmouth, el iniciador del ciclo artúrico. Véase su obra en el manuscrito París, BNF, lat. 4934, ff. 73v-82v.

50 A. Derolez, The Autograph manuscript..., 183. 
intelectuales medievales, es visto como un hito intermedio en la Historia de la Salvación, entre la fundación de la primera ciudad del mundo por Caín, llamada $E_{n o c}{ }^{1}$, y la conquista de Jerusalén. Como ha señalado Jay Rubenstein, estas dos ciudades son puntos de partida y término de la historia humana, desde la ciudad terrena (Enoc) hasta Jerusalén, encarnación terrenal de la ciudad celeste. Pero entremedio la travesía está jalonada por la presencia de otras grandes ciudades (al igual como lo está el Liber floridus): Babilonia, Troya, Roma ${ }^{52}$. La importancia de la urbe como agente histórico queda reflejada también en el capítulo XXVIII dedicado a las grandes ciudades de la antigüedad (De quinque famosis civitatibus), donde Lamberto entrega una reseña de cinco ciudades. En primer lugar Babilonia en Caldea, fundada por el gigante Nimrod y lugar de la cautividad. Luego Babilonia de Egipto (identificada con Menfis en el manuscrito de Wolfenbütte ${ }^{53}$ ) de donde escaparon los judíos bajo la égida de Moisés ${ }^{54}$. En tercer lugar, Ecbatana, escenario de distintos episodios bíblicos. La cuarta ciudad es Nínive, igualmente relevante como espacio de la historia veterotestamentaria. Y por último está Roma, heredera de Troya en el plano histórico-legendario y heredera de las promesas de Israel en el ámbito espiritual. Es decir, son cinco urbes icónicas en la Historia de la Salvación.

\section{La Historia Europea en el Plan del Liber Floridus}

La historia europea se focaliza en Flandes, los francos y el Imperio Germánico. El canónigo de Saint-Omer tiene interés, lógicamente, en realzar la historia local y dar notoriedad a la gesta que la alta nobleza de su región está protagonizando en Tierra Santa, como ha mostrado

51 Lamberto la llama Effrem, siguiendo en este punto a Pseudo Metodio.

52 J. Rubenstein, "Heavenly and Earthly Jerusalem: The View from Twelfth-Century Flanders", en B. KüHNEL y otros (dir.), Visual Constructs of Jerusalem (Brepols, Turnhout 2014) 272-273.

53 W, f. 32v. En el manuscrito de París no se encuentra esta identificación; véase P, f. $47 \mathrm{r}$.

54 Sin embargo, en el texto se dice que esta ciudad fue fundada por el emperador Cambises, es decir, durante la conquista de Egipto por los persas, lo cual es una incoherencia histórica evidente; véase W, f. 32v. 
con abundantes argumentos Jay Rubenstein ${ }^{55}$. De hecho, Lamberto tuvo contacto directo con Bohemundo de Antioquía, uno de los grandes héroes de la Primera Cruzada, cuando este se detuvo en Saint-Omer en su camino de vuelta a Europa el año 1106. De él y su séquito pudo tener noticias de primera fuente sobre los logros de los cristianos en Tierra Santa ${ }^{56}$.

Tanto los Annales (cap. XXII) como las Gesta Romanorum imperatorum (cap. CXXXVII) versan sobre los soberanos del Sacro imperio y las vicisitudes de la porción occidental que toca a Flandes. Este cuadro se completa con las pequeñas historias particulares de otros pueblos germánicos, especialmente los nórdicos: se describen las invasiones vikingas en el norte de Francia y la instalación de los normandos. Esto debe complementarse con un recurso gráfico que Lamberto incluye después del capítulo CLXIX (De Francorum regibus primis), que versa sobre los reyes francos, los emperadores germánicos y los obispos del norte: un mapa de Europa ${ }^{57}$. En esta carta, el primer mapa de Europa a nuestro conocimiento, los nombres de provincias nuevas como Sajonia, Noruega, Baviera, Dacia (Dinamarca), Neustria, Alemania ${ }^{58}$ y Flandes han suplantado a las antiguas denominaciones de la época imperial romana. Además se ha incluido a los eslavos $\left(S_{\text {clavr }}\right)^{59}$. De alguna manera, podría decirse que hay un intento por incluir a todos los pueblos en el horizonte de la historia y de la civilización, como se había hecho ya con los francos, visigodos, lombardos y otras tribus en la época de las primeras invasiones ${ }^{60}$. Los últimos llegados al concierto de pueblos cristianos cierran, por así decir, el elenco de personajes. Todo está ya preparado para la gran función que es el fin del mundo.

55 J. Rubenstein, "Heavenly and Earthly...", 265-276.

56 A. Derolez, "The Abbey of Saint-Bertin, the Liber Floridus and the Origin of the Gesta Francorum Hierusalem expugnantium”, en Manuscripta. A Journal for Manuscript Research 52/1 (2013) 26-27.

57 G, f. 241 r.

58 Entendida como la región de los alamanes.

59 Suecia, no obstante, aparece con la antigua denominación de Scanzia.

60 M. Coumert, Origines des peuples. Les récits du Haut Moyen Áge occidental (550-850) (Institut d'Études Augustiniennes, París 2007) 509-516. 
Para cerrar el cuadro sobre la explotación de textos históricos en el Liber floridus, a los cinco grupos textuales señalados en la Introducción se podría añadir una colección de episodios pequeños, aislados de su contexto histórico y que por ello más bien pasan por cuentos o anécdotas propias de la tradición germánica. Lamberto los saca de diversas fuentes de origen germánico, en su mayoría de los Anales de Saint-Bertin, de los Anales de Lorsch y de la Historia Francorum de Gregorio de Tours. En total forman 15 acápites, que muchas veces no sobrepasan la extensión de un párrafo de pocas líneas ${ }^{61}$. Son pasajes de un tenor peculiar: todos ellos denotan intervenciones sobrenaturales que manifiestan, con signos inequívocos, el vínculo y la cercanía entre Dios y el mundo. Los elementos de la naturaleza juegan aquí un papel de primer orden: los astros, las catástrofes naturales (terremotos, inundaciones, tormentas), incluso el comportamiento de los animales, son portadores de signos divinos ${ }^{62}$. Tres personas fueron fulminadas por un rayo mientras oraban en una iglesia dedicada a san Pedro, en Colonia ${ }^{63}$. Un lobo apareció en la iglesia de san Porcario sembrando gran confusión entre la feligresía reunida ${ }^{64}$. Igualmente sirven como advertencia las hambrunas sobrevenidas en algunas regiones del Imperio Germánico, así como los milagros y las apariciones. Un campesino fue visitado por el Diablo, quien le concedió el poder de adivinar el futuro. Después de probar su arte, obtuvo muchos seguidores que formaron una banda y se pusieron a asaltar los caminos.

${ }^{61}$ Estos capítulos son los siguientes: CXXXIX. Sobre un terremoto y diversos signos; CXLI. Sobre un cierto miembro del Anticristo; CXLII. Sobre los signos en el sol y en la luna y otras cosas; CXLIII. Sobre el paso que surgió como un montículo; CXLIV. Sobre el ayuno de dos doncellas; CXLV. Sobre la cosecha que llovió desde el cielo; CXLVI. Sobre la hambruna, el viento y el ejército; CXLVII. Sobre los tres que fueron muertos por un rayo en Colonia; CXLVIII. Sobre la torre destrozada en Tréveris; CXLIX. Sobre un terremoto en Maguncia y sobre un árbol; CL. Sobre el lobo que se presenta a los senones; CLI. Sobre los signos de la luna y del sol; CLII. Sobre el milagro que ocurrió en Thérouanne; CLIV. La visión de un cierto sacerdote de Inglaterra; CLXXXVI. Sobre un cierto hombre de Antioquía; Visión de Carlos el Calvo, rey de los francos (no listado en el índice; ms G, ff. 207r-208r).

62 I. Draelants, "Le temps dans les textes historiographiques du Moyen Âge", en C. Thomasset - J. Ducos (dir.), Le temps qu'il fait au Moyen Âge. Phénomènes atmosphériques dans la littérature, la pensée scientifique et religieuse (Presses de l'Université de Paris-Sorbonne, París 1998) 122-131.

63 G, f. 211v.

${ }^{64}$ G, f. 211v. 
La turba se disipó solo después de que el caudillo fuera muerto a espada ${ }^{65}$. Un clérigo inglés fue arrebatado en sueños hasta una tierra ignota donde le fue anunciado que si los hombres no hacía penitencia y borraban sus pecados, sobrevendría una densa neblina y al cabo de tres días una hueste de paganos vendría a devastar las tierras a hierro y fuego ${ }^{66}$. Esta visión, tomada de los Annales Bertiniani, año 83967, es una premonición de las depredaciones vikingas, reiteradas a lo largo del siglo IX $^{68}$. Y una vez más, las invasiones de pueblos paganos sedientos de sangre (como antes los godos o los immundi al final) son un anuncio del Apocalipsis.

Las señales del Apocalipsis se amontonan a los ojos de Lamberto. Todo cristiano debe ser capaz de reconocerlas, puesto que Cristo enseñó a sus discípulos a discernir los signos de los tiempos ${ }^{69}$ con los fundamentos necesarios para no dejarse engañar o confundir. Ahora bien, como notaba Verhelst, el canónigo está lejos de concebir un fin de mundo inminente. Aunque el proceso ya ha comenzado (y de ahí la necesidad de recordar sus señales), antes del fin debe ocurrir la conversión de los judíos ${ }^{70}$. De la misma manera, como observa Suzanne Akbari, los judíos no son demonizados en el Liber, sino que son presentados bajo una óptica de esperanza, abiertos a la conversión y a la integración en la comunidad ${ }^{71}$. Una conclusión semejante extrae Jay Rubenstein en un esclarecedor artículo donde analiza los diagramas temporales circulares del Liber floridus. El primero se encuentra en el folio 19r del manuscrito de Gantes y muestra las seis edades del mundo, pero no en esquema lineal, de manera que por su disposición circular, como una rueda, la sexta edad se encuentra con la primera. Lo mismo sucede en el segundo diagrama (folio $19 \mathrm{v}$ ) que representa la sucesión de los reinos universales, en una

65 G, f. 210v.

66 G, f. 216v.

67 A. Derolez, The Making and Meaning..., 146-147.

68 J. Del Hoyo - B. Gazapo (ed.), Anales del Imperio Carolingio. (Años 800-843) (Akal, Madrid 1997) 140.

69 Mt. 16, 1-4; Lc. 12, 54-56.

70 D. Verhelst, "Les textes eschatologiques dans le Liber floridus", en W. VerbeKe y otros (dir.), The Use and Abuse of Eschatology in the Middle Ages (Leuven University Press, Leuven 1988) 301-303.

71 S.C. AkBari, Idols of the East. European Representations of Islam and the Orient, 11001450 (Cornell University Press, Ithaca-London 2009) 89. 
rosa circular en virtud de la cual en el último imperio el poder se topa con el primero volviendo a su origen, en Oriente ${ }^{72}$. No obstante, como explica Rubenstein, si bien la conquista de Jerusalén significa la inauguración de una nueva era, ello no implica necesariamente el fin del mundo ahora. Por eso califica el pensamiento de Lamberto como escatológico y apocalíptico, pero no milenarista ${ }^{73}$. Esta convicción del canónigo puede verse explicitada en el manuscrito autógrafo de Gantes, donde trazó una tabla de anales que fue completando hasta su época, y la dejó en blanco para ser llenada hasta el año 1291, esto es, más de 150 años después de la composición de la obra ${ }^{74}$.

\section{ConCLusión}

Mucho se ha discutido sobre el aparente desorden del material recogido por Lamberto. ¿Cómo encontrarle un sentido a ese caos de relatos y fragmentos? El estudioso francés Bernard Ribémont, simplemente no le ve ninguno. Para él, el autor del Liber no tiene la menor intención de elaborar una propuesta o exponer un sistema, sino simplemente reunir sobre la marcha las cosas que le interesan de la forma más vasta posible ${ }^{75}$. Esto hace que su obra sea una compilación, que se traduce simplemente en reunir un conjunto de extractos (excerpta). Esta tarea se distingue de la transposición, que viene a hacer de la compilación de fragmentos un conjunto inteligible con un objetivo definido. De acuerdo a este esquema, el Liber no sobrepasa el nivel de colección de notas de lectura y no es, concluye Ribémont, una enciclopedia, puesto que no hay tal sin un mínimo de organización del saber ${ }^{76}$. De la misma manera pensaba Yves Lefèvre, para quien Lamberto compuso su obra sin un designio preconcebido, simplemente reuniendo textos que llamaban su atención, y el resultado fue

72 J. Rubenstein, "Lambert of Saint-Omer and the Apocalyptic First Crusade", en N. Paul - S. Yeager (dir.), Remembering the Crusades. Myth, Image, and Identity (The Johns Hopkins University Press, Baltimore 2012) 71-76.

73 J. Rubenstein, "Lambert of Saint-Omer and the Apocalyptic...", 70.

74 G, f. 46r.

75 B. RibÉmont, La «Renaissance» du XIIe siècle et l'Encyclopédisme (Honoré Champion, París 2002) 78 .

76 B. Ribémont, La «Renaissance» du XIIe..., 81-82. 
una "vitrina de coleccionista"77. Albert Derolez, en cambio, sí considera que se trata de una enciclopedia, pero una enciclopedia metafísica, que no se interesa por los temas tradicionales del género (descripción del mundo, división de las ciencias, tratado del tiempo ${ }^{78}$. Pero, no obstante el caos aparente en el Liber, hemos observado una tendencia muy clara a desarrollar tres áreas específicas del saber: la teología, la historia y la cosmografía, lo que nos muestra de entrada una intención determinada del autor, que se traduce en un objetivo bien definido y en un plan previamente concebido en función de aquél. Esto nos habla además de una labor consciente en la selección de fragmentos para ser incluidos en la obra. Limitado lógicamente a los tratados disponibles en las bibliotecas locales, Lamberto escogió cuidadosamente los extractos y los colocó de manera estratégica de manera que, apoyándose unos en otros y propiciando el diálogo entre sí, formaran una trama coherente, necesaria para la ejecución del proyecto redaccional y para la comunicación del mensaje escatológico.

Conocer a Dios y su plan de salvación a través de las cosas creadas, he aquí el objetivo último del Liber floridus. Por ello el interés por la constitución física del cosmos, que lo lleva a extractar numerosos capítulos sobre el tema. Lamberto observa, describe, pero guarda las distancias. No se refiere, por ejemplo, a la posible influencia de los astros en las personas, algo que se hacía cada vez más frecuente en aquella época, debido a la reciente traducción de textos árabes al latín. En el capítulo LXXIX desarrolla una discusión sobre las constelaciones, tomada de un manuscrito Aratea, una copia ilustrada de los Phaenomena del astrónomo griego Arato ( $†$ 240/39 a.C.), pero omite cualquier consideración de carácter astrológico ${ }^{79}$. Antes bien, el esquema apocalíptico-cosmográfico del capítulo LXXVIII (De IIII elementis) ${ }^{80}$ muestra claramente la subordinación de los cuatro elementos a la majestad divina, y que por lo mismo serán transfigurados al final

77 Y. LeFÈVRE, "Le Liber Floridus et la littérature encyclopédique au moyen âge", en A. Derolez (dir.), Liber floridus colloqvivm. Papers read at the international meeting beld in the University Library Ghent on 3-5 september 1967, (E. Story-Scientia, Gantes 1973) 8-9.

78 A. Derolez, The Autograph manuscript..., 181-183.

79 C. Heitzmann, Die Sterne lïgen nicht. Astrologie und Astronomie im Mittelalter und der Früben Neuzeut (Harrassowitz Verlag, Wiesbaden 2008) 20.

80 G, f. 88r. 
de los tiempos, lo que viene reforzado por los textos explicativos que acompañan al esquema: "En el principio mismo de la Creación fueron hechos de la nada el cielo, la tierra y los ángeles, el aire y el agua" ${ }^{\prime 1}$.

El medio que rodea al ser humano en la superficie terrestre presenta la misma conexión con la divinidad y es, por tanto, objeto de las consideraciones escatológicas. No podía ser de otro modo en el pensamiento de Lamberto, dado que el espacio geográfico es el escenario donde se desarrolla el drama de la humanidad. Por eso el mapamundi más importante de su enciclopedia lleva por título "Hormista regnorum mundi" Según Leonid Chekin, la palabra (b)ormista tiene el sentido de mappa o exposición, y refleja la unidad entre la historia y la geografía, con un fuerte sentido moral y una connotación escatológica ${ }^{83}$. Pero los mapamundis del Liber floridus están igualmente conectados intrínsecamente con la ciencia histórica, algo muy propio del saber enciclopédico. Esta idea tenía su origen en la obra del sacerdote hispano Paulo Orosio (siglo V) que unió historia con scientia locorum ${ }^{84}$. $\mathrm{Y}$ así el gran mapamundi espacializa no pocos de los elementos de carácter escatológico: el Paraíso terrenal, la morada de Enoc y Elías, Babilonia, el valle cerrado de Gog y Magog, y diversas razas monstruosas ${ }^{85}$.

El análisis de los textos históricos del Liber floridus nos ha mostrado que Lamberto de Saint-Omer tenía plena conciencia del valor de la Historia, en cuanto manifestación de la providencia divina. El tenor de los fragmentos y su disposición en la obra le permiten exhibir a sus contemporáneos el designio de Dios para los hombres, que incluye momentos estelares

81 G, f. 88r: "In ipso quidem principio conditionis facta sunt cęlum, terram, angeli, aer et aqua de nichilo".

82 G, f. 69v.

83 L. Chekin, Northern Eurasia in Medieval Cartography. Inventory, Text, Translation, and Commentary (Brepols. Turnhout 2006) 187, nota 4.

84 A. Hiatt, "Worlds in books", en E. Steiner - L. Ransom (dir.), Taxonomies of Knowledge. Information and Order in Medieval Manuscripts (University of Pennsylvania Press, Philadelphia 2015) 37.

85 De hecho, el trabajo de Danielle Lecoq sobre los numerosos mapamundis del Liber floridus y las reflexiones de Jean-Marie de Smet sobre el corpus astronómico, muestran la misma conexión con la escatología. Véase D. LECOQ, "La Mappemonde du...", 25-28; 34-37 ; J.-M. DE SMET, "La mentalité religieuse du chanoine Lambert", en A. Derolez (dir.), Liber floridus colloqvivm..., 12. 
desde la creación, la caída, la redención y la vuelta a la casa del Padre, de la mano de seres de carne y hueso, con vicios y virtudes. Porque no solo son relevantes los santos y las personalidades de la Iglesia, sino también Alejandro Magno, los emperadores romanos, los guerreros germánicos, los caballeros que partieron a las cruzadas... Para entender el universo en su conjunto (y esto incluye su destino final), la historia en su totalidad aporta una mirada preciosa que complementa a la teología. Lamberto se cuida bien de no usar la historia para predecir el futuro (de hecho, en la Edad Media, nadie predice el futuro, porque este ya está escrito en la Biblia), pero sí le encuentra una gran utilidad para comprender la relación entre Dios y el mundo.

Historia y cosmografía forman, pues, junto con la reflexión teológica, un sistema único y complejo, en el cual tanto la naturaleza como el ser humano, creado y por tanto un elemento también natural en ese sistema, contribuyen a su desarrollo y culminación, bajo la guía de la Providencia. Algunas décadas después, la escolástica ordenará la revelación divina a partir de la naturaleza creada y la Biblia (revelación natural y revelación sobrenatural). Lamberto no hace teología propiamente hablando, pero en cierta forma es consciente de esto al construir su obra a base de textos cosmográficos y teológicos. Y agrega además la historia a ese corpus, no tanto como una tercera vía para el conocimiento de Dios, eso sería ir muy lejos, pero sí como un elemento que, leído en clave anagógica, es provechoso para entender el universo en su sentido más profundo y la acción de su Creador. De alguna manera, Lamberto se convierte en precursor de los sabios del siglo XIII que usaron la cosmografía de los griegos para desarrollar la teología cristiana. En su época, la Física de Aristóteles ciertamente aún no había sido traducida al latín, pero eso no le impidió utilizar el comentario de Calcidio al Timeo, los Phaenomena de Arato y otras obras clásicas al servicio de la teología.

En definitiva, Lamberto logró entrelazar la historia, la cosmografía y la teología para construir un discurso explicativo relativamente integrado. Sólo así se comprende a cabalidad el título que escogió para su enciclopedia (tomado del nombre Flores) y su tremenda apertura hacia todos los campos del saber, que es precisamente lo que permite a los lectores saborear tanto las cosas del cielo como las de la tierra, como queda reflejado en el prólogo: 
Yo, Lamberto hijo de Onulfo, canónico de Saint-Omer, compuse este librito con flores de diversos autores en honor de Dios y de san Audomaro nuestro patrono, para que las fieles abejillas confluyan a él como a un prado celestial adornado de diversas flores, y beban de ahí la dulzura del sabor celestial. Porque así lo requiere el orden, lo intitulé Florido, porque florece con los adornos de diversos libros y gana en solidez con la narración de cosas admirables ${ }^{86}$.

El prado adornado de flores es la imagen perfecta de la armonía, de esa armonía que invita a elevar el alma a Dios y alabarlo en la naturaleza. Aunque Isidoro en sus Etimologías ya había notado la profunda imbricación entre tiempo y espacio - entre historia y mundo natural-es Lamberto quien, varios siglos después, vuelve a poner énfasis en esta relación. El Liber floridus presenta el conjunto de la creación como speculum o espejo del cosmos, integrando a un tiempo la descripción de la naturaleza y la narración de los sucesos históricos, y donde el producto final, es decir, el compendio del conocimiento, refleja la majestad y la inteligencia ordenadora de Dios. Podemos concluir que Lamberto de Saint-Omer propone una forma novedosa de organización del saber e inaugura así la era de las enciclopedias especulares medievales, género que alcanzó un gran desarrollo en Occidente de lo cual dan testimonio las obras de Honorio Agustodunense, Ricardo de Saint-Victor, Herrade de Landsberg, Alejandro Neckam y un largo etcétera de enciclopedistas posteriores.

86 G, f. 3v: "Ego Lambertus filius Onulfi, canonicus Sancti Audomari, libellum istum de diversorum auctorum floribus Deo sanctoque Audomaro pro patrono nostro contexui, ut tamquam de cęlesti prato flore diverso coadunato, fideles apiculę ad hunc confluerent, saporisque cęlestis inde dulcedinem haurirent. Quem, quoniam sic ratio postulat, Floridum intitulavi, quia et variorum librorum ornatibus floret rerumque mirandarum narratione prepollet". 